\title{
COMPLETELY REGULAR MAPPINGS AND DIMENSION ${ }^{1}$
}

\author{
BY DAVID C. WILSON
}

Communicated by Shlomo Sternberg, March 18, 1970

1. Introduction. In an earlier paper [12] the author proved the following theorem: There exists a monotone open map of the universal curve onto any continuous curve such that each point-inverse set is also a universal curve. Since these mappings are open and have homeomorphic point-inverse sets, it is natural to ask whether or not these mappings are completely regular. Theorem 1 of this paper shows that they will be completely regular only if the range is a point. Theorem 1, Theorem 3 , and the corollary to Theorem 3 all give conditions on completely regular mappings so that they will not raise dimension. Theorem 4 actually classifies completely regular mappings of a certain type.

The author wishes to express his gratitude to Professors G. Bredon and D. Erle for their assistance and helpful suggestions in the preparation of this paper.

\section{The main theorem.}

THEOREM 1. If $f$ is a completely regular mapping of an n-dimensional compactum $X$ onto a compactum $Y$ and $\check{H}^{n}\left(f^{-1}(y)\right) \neq 0$ for all $y \in Y$, then $Y$ is 0-dimensional.

Lemma 1. Let $X$ be an $n$-dimensional compactum. Let $J$ be a finite polyhedron contained in $E^{2 n+1}$ of dimension less than $n+1$. If $f$ is a mapping of $X$ into $E^{2 n+1}$ and $\eta>0$, then there exists a homeomorphism $h: X \rightarrow E^{2 n+1}$ such that $d(f, h)<\eta$ and $h(X) \cap J=\varnothing$.

Proof of Lemma 1. Approximate $f$ by a mapping $g$ whose range is contained in an $n$-polyhedron which (by general positioning) misses $J$. Since the set of homeomorphisms is dense in the function space $\left(E^{2 n+1}\right)^{X}$, we can find a homeomorphism $h$ which approximates $g$ and such that $h(X) \cap J=\varnothing$.

The homology theory in this paper will be singular homology with integer coefficients. If $J$ is a singular $n$-cycle, then $|J|$ will denote its

AMS 1969 subject classifications. Primary 5470, 5538; Secondary 5460.

Key words and phrases. Completely regular mappings, finite dimensional compacta, Alexander duality, Vietoris mapping theorem.

1 This research was supported in part by the National Science Foundation, under NSF grant GP-7952X1. 
carrier. The cohomology theory will be Cech cohomology with integer coefficients.

Lemma 2. Let $M$ and $N$ be compact subsets of $E^{2 n+1}$ and let $J$ be an $n$-cycle which represents a class in $H_{n}\left(E^{2 n+1}-M\right)$. If there exists a homotopy equivalence $h$ of $M$ into $N$ which moves no point of $M$ more than $\frac{1}{2} d(M,|J|)$, then $J$ represents a nonzero class in $H_{n}\left(E^{2 n+1}-M\right)$ if and only if $J$ represents a nonzero class in $H_{n}\left(E^{2 n+1}-N\right)$.

Proof. Let $U$ be an open subset of $E^{2 n+1}$ such that $|J| \subseteq U$ $\subseteq E^{2 n+1}-(M \cup N), E^{2 n+1}-U$ is compact, and $d(U, M)>\frac{1}{2} d(M,|J|)$.

If $i_{1}$ and $i_{2}$ denote inclusion mappings, then the above restrictions insure that the following diagram is homotopy commutative:

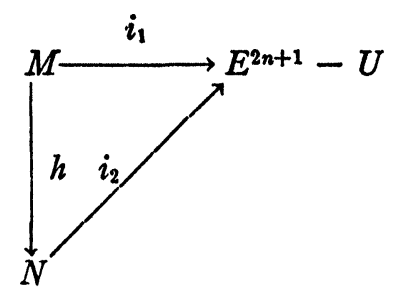

Thus, $i_{1}^{*}=h^{*} \circ i_{2}^{*}$.

From the naturality of Alexander Duality, we get the following commutative diagram:

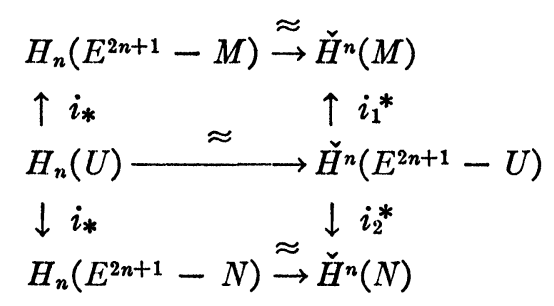

Since $i_{1}^{*}=h^{*} \circ i_{2}^{*}$ and $h^{*}$ is an isomorphism, the result follows.

Lemma 3. Let $A$ and $B$ be disjoint closed subsets of an n-dimensional compactum $X$. If $\check{H}^{n}(A) \neq 0$, then there exists an imbedding $h: X \rightarrow E^{2 n+1}$ and $n$-cycle $J$ such that $h(X) \cap|J|=\varnothing$ and $J$ represents a nonzero class in $H_{n}\left(E^{2 n+1}-h(A)\right)$ and the zero class in $H_{n}\left(E^{2 n+1}-h(B)\right)$.

Proof. Let $f_{1}$ be an imbedding of $A$ into $E^{2 n+1}$ and let $J$ be a simplicial $n$-cycle which represents a nonzero class in $H_{n}\left(E^{2 n+1}-f_{1}(A)\right)$. By Tietze's extension theorem, we can find a map $f$ from $X$ into $E^{2 n+1}$ which extends $f_{1}$ and such that $f(B)$ is outside some ball $N$ con- 
taining $f_{1}(A) \cup|J|$. By Lemma 1 there exists a homeomorphism $h$ of $X$ into $E^{2 n+1}$ such that

$$
d(f, h)<\min \left\{d(f(B), N), \frac{1}{2} d(|J|, f(A))\right\}
$$

and $h(X) \cap|J|=\varnothing$. By Lemma $2, J$ represents a nonzero class in $H_{n}\left(E^{2 n+1}-h(A)\right)$.

Note that in the proof of Lemma 3 we did not try to extend the imbedding $f_{1}$ to $h$, but rather "moved" it slightly. This is neceIsary since the examples in [2] can be used to show that there exists an imbedding of a Cantor set plus a circle into $E^{n}, n \geqq 5$, which cannot be extended to a Cantor set plus a disk.

Proof of Theorem 1. Since $Y$ is compact, it is sufficient to show that each component $K$ of $Y$ is a point.

Suppose $K$ contains two distinct points $y_{1}$ and $y_{2}$. By Lemma 3 we can find an imbedding $h$ of $X$ into $E^{2 n+1}$ and $n$-cycle $J$ such that $h(X) \cap|J|=\varnothing$ and the class $[J]$ is nonzero in $H_{n}\left(E^{2 n+1}-h\left(f^{-1}\left(y_{1}\right)\right)\right)$ and $[J]=0$ in $H_{n}\left(E^{2 n+1}-h\left(f^{-1}\left(y_{2}\right)\right)\right)$.

Let $Y_{1}=\left\{y \in K:[J] \neq 0\right.$ in $\left.H_{n}\left(E^{2 n+1}-h\left(f^{-1}(y)\right)\right)\right\}$ and $Y_{2}$ $=\left\{y \in K:[J]=0\right.$ in $\left.H_{n}\left(E^{2 n+1}-h\left(f^{-1}(y)\right)\right)\right\}$. Note that $y_{1} \in Y_{1}$ and $y_{2} \in Y_{2}$. Using Lemma 2 together with the complete regularity of $f$ it is easy to show that both $Y_{1}$ and $Y_{2}$ are open. Since $K=Y_{1} \cup Y_{2}$, we have a contradiction of the assumption that $K$ is connected. Thus, $K$ is a point.

REMARK. Note in Theorem 1 that if $Y$ is connected, then $Y$ is a point.

3. Mappings which do not raise dimension. The next theorem is a cohomology analogue of Theorem 1 of [4].

TheOREM 2. If $f$ is a monotone mapping of an n-dimensional compactum $X$ onto a finite dimensional compactum $Y$ and $H^{k}\left(f^{-1}(y)\right)=0$ for all $k \geqq 1$ and all $y \in Y$, then

$$
\operatorname{dim} Y \leqq n \leqq \operatorname{dim} Y+\sup \left\{\operatorname{dim} f^{-1}(y)\right\} .
$$

PRoof. The second inequality follows from Theorem VI of [8].

To show the first inequality we will use the characterization of dimension given in Theorem VIII 2 of [10]. Thus, it is sufficient to show that if $C$ is a closed subset of $Y$ and $m \geqq n$, then the homomorphism $i^{*}$, induced by the inclusion $i: C \rightarrow Y$, is onto. Since $\operatorname{dim} X=n$, we have the following commutative diagram:

$$
\begin{array}{r}
0 \leftarrow \check{H}^{m}\left(f^{-1}(C)\right) \leftarrow \check{H}^{m}(X) \\
\uparrow f^{*} \quad i^{*} \check{\imath}^{*} \\
\check{H}^{m}(C) \stackrel{\check{H}^{m}(Y)}{\longleftarrow}
\end{array}
$$


By the Vietoris Mapping Theorem we know that $f^{*}$ is an isomorphism so that $i^{*}$ is onto. Thus, $\operatorname{dim} Y \leqq n$.

THEOREM 3. Let $f$ be a completely regular mapping of an n-dimensional compactum $X$ onto a finite dimensional compactum $Y$. If for each $y \in Y f^{-1}(y)$ has only a finite number of components and $\check{H}^{k}\left(f^{-1}(y)\right)=0$ for $k=1, \cdots, n-1$, then $\operatorname{dim} Y \leqq \operatorname{dim} X$.

Proof. It is sufficient to show that each component $K$ of $Y$ has dimension $\leqq \operatorname{dim} X$.

If $\check{H}^{n}\left(f^{-1}(y)\right) \neq 0$ for some $y \in K$, then, by Theorem $1, K$ is a point.

If $\breve{H}^{n}\left(f^{-1}(y)\right)=0$ for all $y \in K$, then factor $f$ by the monotone light factorization theorem:

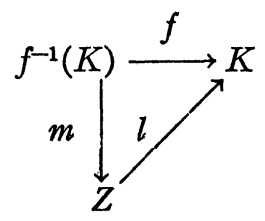

Since $l$ is a finite to one open mapping, we know that $\operatorname{dim} Z$ $=\operatorname{dim} K<\infty$ [9]. Hence, by applying Theorem 2 to the map $m$, we know that $\operatorname{dim} K=\operatorname{dim} Z \leqq \operatorname{dim} X$.

COROLlARY. If $f$ is a completely regular mapping of a 1-dimensional compactum $X$ onto a finite dimensional compactum $Y$ such that each point-inverse has a finite number of components, then $\operatorname{dim} Y \leqq 1$.

Remark. Note that the above corollary is not true if we allow the point-inverse sets to be cantor sets. For Theorem 2 of [12] states that there exists a light open mapping of the universal curve onto any nondegenerate continuous curve such that each point-inverse set is a Cantor set. Note that these mappings will be completely regular.

ExAmple. If $Y$ is any continuous curve, then there exists a 2-dimensional continuum $X$ and a monotone completely regular mapping $F$ of $X$ onto $Y$.

Let $\sigma^{7}$ denote the standard 7 -simplex. If $A, B C \sigma^{7}$, then let $A \cdot B$ $=\left\{x \in \sigma^{7}: x \in\langle a, b\rangle, a \in A, b \in B\right\}$. Let $\sigma_{1}^{3}$ and $\sigma_{2}^{3}$ denote two disjoint 3 -simplices which are faces of $\sigma^{7}$. Let $u_{1}$ and $u_{2}$ be two copies of the universal curve in $\sigma_{1}^{3}$ and $\sigma_{2}^{3}$, respectively. Let $f_{i}: U_{i} \rightarrow Y$ be completely regular mappings of the type in the above remark. Let $X$ $=\left\{f_{1}^{-1}(y) \cdot f_{2}^{-1}(y): y \in Y\right\}$. There is a natural monotone completely regular mapping $F$ of $X$ onto $Y$ which extends each $f_{i}$. It can be shown 
that $\operatorname{dim} F^{-1}(y)=1$ and $\operatorname{dim} X=2$. Note that this example follows the technique of [7].

THEOREM 4. If $f$ is a completely regular mapping of a 1-dimensional compactum $X$ onto a finite dimensional continuum $Y$ such that $f^{-1}(y)$ is a continuous curve for all $y \in Y$, then either $Y$ is a point or $Y$ is homeomorphic to $X$ under $f$.

PRoOF. If $f^{-1}(y)$ contains a simple closed curve, then by Theorem 1 we know that $Y$ is a point.

Suppose $f^{-1}(y)$ contains no simple closed curve for all $y \in Y$. If $f^{-1}(y)$ and $Y$ are both nondegenerate, then by Theorem 3 of [4] we know that $\operatorname{dim} X=\operatorname{dim} Y+1 \geqq 2$. This contradicts the assumption that $X$ is 1 -dimensional.

\section{REFERENCES}

1. R. D. Anderson, A characterization of the universal curve and a proof of its homogeneity, Ann. of Math. (2) 67 (1958), 313-324. MR 20 \#2675.

2. W. A. Blankinship, Generalization of a construction of Antoine, Ann. of Math. (2) 53 (1951), 276-297. MR 12, 730.

3. A. Dold, (Co-)homology properties of topological manifolds, Conference on the Topology of Manifolds, Prindle, Weber, and Schmidt, 1968, pp. 47-58.

4. E. Dyer, Certain transformations which lower dimension, Ann. of Math. (2) 63 (1956), 15-19. MR 17, 993.

5. —, Regular mappings and dimension, Ann. of Math. (2) 67 (1958), 119-149. MR 19, 1071.

6. E. Dyer and M. E. Hamstrom, Completely regular mappings, Fund. Math. 45 (1958), 103-118. MR 19, 1187.

7. W. Hurewicz, Über oberhalb-stetge Zerlegungen von Punktmengen in Kontinua, Fund. Math. 15 (1930), 57-60.

8. W. Hurewicz and H. Wallman, Dimension theory, Princeton Math. Series, vol. 4, Princeton Univ. Press, Princeton, N. J., 1941. MR 3, 312.

9. K. Nagami, Mappings of finite order and dimension theory, Japan J. Math. 30 (1960), 25-54. MR $25 \# 5494$.

10. J. Nagata, Modern dimension theory, Bibliotheca Math., vol. 4, Interscience, New York, 1965. MR 34 \#8380.

11. E. Spanier, Algebraic topology, McGraw-Hill, New York, 1966. MR 35 \#1007.

12. D. C. Wilson, Open mappings of the universal curve onto continuous curves (to appear).

Institute for Advanced Study, Princeton, New Jersey 08540 